\title{
3-D Super Resolution Imaging using a 62+62 Elements Row-Column Array
}

Jensen, Jørgen Arendt; Schou, Mikkel; Ommen, Martin Lind; Øygard, Sigrid Husebø; Sams, Thomas; Stuart, Matthias Bo; Thomsen, Erik Vilain; Larsen, Niels Bent; Beers, Christopher; Tomov, Borislav Gueorguiev

Published in:

Proceedings of 2019 IEEE International Ultrasonics Symposium

Link to article, DOI:

10.1109/ULTSYM.2019.8926092

Publication date:

2019

Document Version

Peer reviewed version

Link back to DTU Orbit

Citation (APA):

Jensen, J. A., Schou, M., Ommen, M. L., Øygard, S. H., Sams, T., Stuart, M. B., Thomsen, E. V., Larsen, N. B., Beers, C., \& Tomov, B. G. (2019). 3-D Super Resolution Imaging using a 62+62 Elements Row-Column Array. In Proceedings of 2019 IEEE International Ultrasonics Symposium (pp. 1838-1841). IEEE.

https://doi.org/10.1109/ULTSYM.2019.8926092

\section{General rights}

Copyright and moral rights for the publications made accessible in the public portal are retained by the authors and/or other copyright owners and it is a condition of accessing publications that users recognise and abide by the legal requirements associated with these rights.

- Users may download and print one copy of any publication from the public portal for the purpose of private study or research.

- You may not further distribute the material or use it for any profit-making activity or commercial gain

- You may freely distribute the URL identifying the publication in the public portal 


\title{
3-D Super Resolution Imaging using a 62+62 Elements Row-Column Array
}

\author{
Jørgen Arendt Jensen ${ }^{1}$, Mikkel Schou ${ }^{1}$, Martin Lind Ommen ${ }^{1}$, Sigrid Husebø Øygard ${ }^{1}$, \\ Thomas Sams ${ }^{1}$, Matthias Bo Stuart ${ }^{1}$, Erik Vilain Thomsen ${ }^{1}$, \\ Niels Bent Larsen ${ }^{1}$, Christopher Beers ${ }^{2}$ and Borislav Gueorguiev Tomov ${ }^{1}$ \\ ${ }^{1}$ Department of Health Technology, \\ Technical University of Denmark, DK-2800 Lyngby, Denmark \\ ${ }^{2}$ BK Medical, 401 Science Park Road, State College, PA 16803, USA
}

\begin{abstract}
Current 2-D Super Resolution (SR) imaging is limited by the slice thickness determined by the elevation focus. The fixed, geometric elevation focus is often poor due to its high F-number. SR images are, thus, a summation of vessels across the elevation plane without the possibility to track scatterers in 3-D for full visualization. 3-D SR imaging has been obtained by translating the probe, but this does not remove the elevation summation. Full 3-D can be acquired using 2-D matrix probes, but the equipment is expensive, and the amount of data is excessive, when channel data are acquired over thousands of elements for minutes. This paper demonstrates that full volumetric SRI can be attained using a 62+62 channels Row-Column (RC) probe with a high frame rate and with $\mu \mathrm{m}$ precision. Data were acquired by a $3 \mathrm{MHz} 62+62 \mathrm{PZT} \mathrm{RC}$ probe with $\lambda / 2$ pitch connected to the SARUS scanner. A synthetic aperture scan sequence with 32 positive and 32 negative emissions was employed for pulse inversion (PI) imaging with an MI of 0.3 . The pulse repetition frequency was $10 \mathrm{kHz}$ for a $156 \mathrm{~Hz}$ volume rate. A PEGDA $700 \mathrm{~g} / \mathrm{mol}$ based hydrogel flow-microphantom was 3-D printed by stereo-lithography. It contains a single cylindrical $200 \mu \mathrm{m}$ diameter channel placed $3 \mathrm{~mm}$ from the top surface of the phantom. After a $5.8 \mathrm{~mm}$ long inlet, the channel bends $90^{\circ}$ into a $7 \mathrm{~mm}$ long central region before bending $90^{\circ}$ again into the $5.8 \mathrm{~mm}$ outlet. The flow channel was infused at $1.61 \mu \mathrm{L} / \mathrm{s}$ with Sonovue (Bracco) in a 1:10 dilution. The received RF signals from the 62 row elements were beamformed with PI to yield a full volume of $15 \times 15 \times 15 \mathrm{~mm}^{3}$. The interpolated 3-D positions of the bubbles were estimated after local maximum detection. The reconstructed 3-D SR volume clearly shows the $200 \mu \mathrm{m}$ channel shape with a high resolution in all three dimensions. The center line for the channel was found by fitting a line to all bubble positions, and their radial position calculated. The observed fraction of bubbles falling outside the channel was used for estimating the location precision. The precision was $16.5 \mu \mathrm{m}$ in the $y-z$ plane and $23.0 \mu \mathrm{m}$ in the $x-z$ plane. The point spread function had a size of $0.58 \times 1.05 \times 0.31 \mathrm{~mm}^{3}$, so the interrogated volume was 15,700 times smaller than for normal volumetric B-mode imaging. This demonstrates that full 3-D SRI can be attained with just 62 receive channels. The $S A$ sequence has a low MI, but attains a large measured penetration depth of $14 \mathrm{~cm}$ in a tissue mimicking phantom, due to the large $\mathrm{RC}$ probe size. The $156 \mathrm{~Hz}$ volume rate also makes it possible to track high velocities in 3-D in the volume.
\end{abstract}

\section{INTRODUCTION}

Super resolution (SR) imaging has recently been introduced in ultrasound. The method is based on tracking the centroid of contrast agent bubbles and thereby paint an image of the micro-vasculature [1-6]. Very high resolutions can be attained from the non-linear estimation of the target's centroid positions, and reports of image resolution in the $10 \mu \mathrm{m}$ range have been given [7]. The results presented are predominantly in 2-D, and the high resolution is only attained in the image plane. The out-of-plane resolution is determined by the elevation focus, which often is poor due to the fixed-focus lens. The F-number (imaging depth divided by the probe width) is usually 2 to 5 giving an ideal resolution of $2 \lambda-5 \lambda$ at the focal depth and worse away from it ( $\lambda$ is the wavelength given by $c / f_{0}$, where $c$ is the speed of sound and $f_{0}$ is transducer center frequency). The images are acquired over several seconds to minutes generating Gbytes of data. Currently, most SRI is conducted using 1-D array probes due to the large amount of data generated, and that few scanners are capable of full 3-D imaging.

Visualization of 3-D SR volumes has been performed by several groups using mechanically translated linear array probes $[6,8,9]$, but such a setup does not make it possible to estimate the out-of-plane location. SR has also been made using two orthogonal probes for 3-D localization in a line [10], and mechanical scanning is needed to cover a full volume. A matrix probe is, thus, needed for avoiding mechanical scanning.

Currently, the largest research scanners have 1024 channels [11, 12], and they generate around 20-50 Gbytes/s of data for $3 \mathrm{MHz}$ probes, only making short acquisitions possible and precluding the use of high-frequency probes. They can handle 2 -D arrays with $32 \times 32=1024=N^{2}$ elements, which have been fabricated with $\lambda / 2$ pitch. This makes them suitable for phased array imaging, but severely limits their focusing ability due to their small size and hence high F-numbers.

The problem can be somewhat alleviated by using sparse arrays, and Harput et al. [13] recently used a 512 elements sparse 2-D array based on a spiral pattern to acquire full 3-D SR imaging. Two 256 channels research scanners [14] were used for scanning of $200 \mu \mathrm{m}$ cellulose tubes with a final localization precision of $18 \mu \mathrm{m}$. The main drawback of this approach is the many transducer channels needed to avoid grating lobes and the corresponding large amounts of data generated per second. Further, the probe is quite small 
( $\varnothing 10.4 \mathrm{~mm}$ ), as it has to be nearly fully populated to avoid side and grating lobes, limiting the possible F-numbers.

This paper describes a 3-D SR method based on a RowColumn (RC) array with only 62+62 elements. The approach is implemented using a prototype RC array, and the imaging is conducted using the SARUS research scanner [11]. Its precision is investigated using a 3-D printed micro-phantom and is estimated from the located bubbles in the phantom.

\section{Methods}

\section{A. Data acquisition and beamforming}

A prototype $3 \mathrm{MHz}$ PZT RC array with 62 rows and 62 columns was used for the data acquisition [15]. It contains amplifiers in the handle and was fabricated with edge apodization to reduce ghost echoes after the main point spread function (PSF) [16]. The probe has $\lambda / 2$ pitch to avoid grating lobes. It was connected to the SARUS scanner [11], which acquired full RF data for all the receiving channels.

A synthetic aperture, pulse inversion sequence was used for imaging. Transmissions were conducted using the rows, and data were received on all 62 columns. The virtual line sources emitted cylindrical waves [17] in a sequence with 32 positive emissions and 32 negative emission to make pulse inversion imaging possible. The transmit F-number was -1 using 32 Hanning apodized active elements, with the virtual source placed behind the array.

\section{B. 3-D micro-phantom}

A flow micro-phantom is fabricated for validating the approach by 3-D printing of a PEGDA $700 \mathrm{~g} / \mathrm{mol}$ hydrogel using stereo-lithography, as described in [18]. The phantom measures $21.1 \times 8.16 \times 11.9 \mathrm{~mm}^{3}$, and the voxel size of the printer is $(\Delta x, \Delta y, \Delta z)=10.8 \times 10.8 \times 20 \mu \mathrm{m}^{3}$. The flow microphantom contains a single cylindrical $100 \mu \mathrm{m}$ radius channel placed $3 \mathrm{~mm}$ from the top surface of the phantom. After a 5.8 $\mathrm{mm}$ long inlet, the channel bends $90^{\circ}$ into a $7 \mathrm{~mm}$ long central region before bending $90^{\circ}$ again into the $5.8 \mathrm{~mm}$ outlet. The flow channel is infused at $1.61 \mu \mathrm{L} / \mathrm{s}$ with SonoVue (Bracco, Milano, Italy) in a 1:10 dilution, giving a peak velocity of $102.4 \mathrm{~mm} / \mathrm{s}$.

\section{Processing pipeline}

The beamformed volumes are processed in Matlab using our 3-D SR processing pipeline consisting of three steps. The first is to beamform the stored RF data from the SARUS scanner using the beamforming strategy described by Rasmussen et al. $[16,17]$ implemented in Matlab and running on an Nvidia GeForce GTX 1050 Ti (Nvidia, Santa Clara, CA, USA) GPU [19]. For the flow micro-phantom the second harmonic signal is employed, and a filter matched to the second harmonic is employed on all the received signals. The GPU beamformer was used for making the focusing of the full volumes for all emissions with an F-number of 1.5 in transmit and 1 in receive with a dynamic Hanning apodization weighting the elements. The volumes with a size of $\pm 15 \lambda$ in both the $x$ and $y$ directions were beamformed with a line density of $\lambda / 2$ covering the full depth of the phantom. The sampling density in the $z$ direction is $\lambda / 16$. All emissions are added to generate the high resolution volume (HRV), and the positive and negative emissions HRVs are added to enhance the bubble signals.

The second step is to subtract the stationary background signal. The mean value of twenty volumes is found and subtracted from all the 400 volumes acquired. The envelope of the HRV is then found using a Hilbert transform and log compressed to a $40 \mathrm{~dB}$ dynamic range in relation to the data in the volume for finding locations.

The bubble locations can either be found from calculation of the centroid of local maxima, or the peak locations can be interpolated to increase the location accuracy. Experimentation with the data showed that the interpolation scheme is the most stable and accurate method, and this is the one used in this paper.

The third stage finds bubble locations by interpolating the peak position by fitting a second order polynomial to the data and then finding its interpolated maximum position $x_{i}$, as:

$$
x_{i}=i-\frac{0.5(d(i+1, j, k)-d(i-1, j, k))}{d(i+1, j, k)-2 d(i, j, k)+d(i-1, j, k)},
$$

where $i, j, k$ are the indices of the maximum and $d$ is the envelope data for the volume. This is conducted in all three coordinates $x_{i}, y_{j}, z_{k}$ with similar equations for an increased resolution in all three directions.

\section{Statistical evaluation}

The detected bubble locations are randomly distributed in the flow micro-phantom tube due to noise in the localization estimation, and some of them will appear to be located outside the phantom wall. The distribution of positions found can therefore yield an estimate of the localization precision. An estimate of the $y-z$ and $x-z$ precision can be obtained from the two straight segments of the $200 \mu \mathrm{m}$ channel phantom. In the straight segments a line is fitted to the data and considered an estimate of the center of the channel, and the distance from each bubble to the center is calculated. Assuming the measurement uncertainty in each dimension is normal distributed, the radial distribution of all bubbles in the segment will follow the distribution

$$
f(r)=2 \pi r \int_{\left|\vec{r}_{t}\right|<R} \frac{1}{\pi R^{2}} \frac{1}{2 \pi \sigma^{2}} \exp \left(\frac{-\left|\vec{r}-\vec{r}_{t}\right|^{2}}{2 \sigma^{2}}\right) d^{2} r_{t},
$$

where $r$ is radial position, $R$ is the radius of the tube, and $\sigma$ is the standard deviation of the uncertainty. The integral is a convolution of a constant density $\left(1 /\left(\pi R^{2}\right)\right)$ with a two-dimensional Gaussian. The non-analytical integral (2) is estimated in a Monte-Carlo calculation and is a Rayleigh distribution convolved with a uniform disk distribution of radius $R=100 \mu \mathrm{m}$. The fraction of bubbles estimated to fall outside the tube can then be translated into an estimate for the standard deviation $\sigma$ (localization precision). 


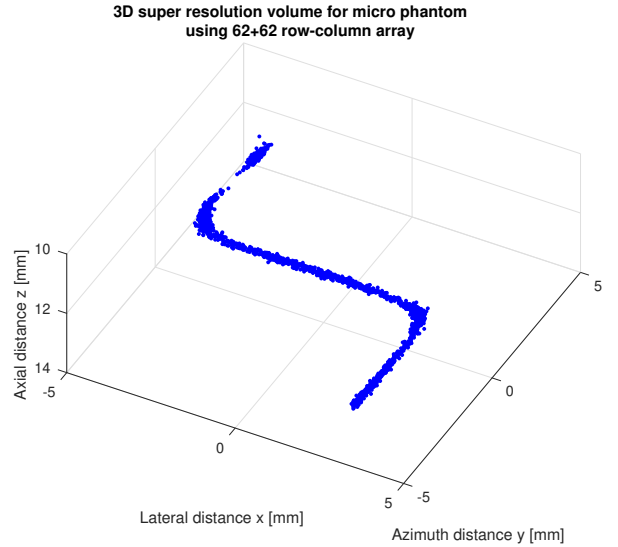

Fig. 1. Visualization of the 3-D phantom after detection of bubble locations each indicated by a blue dot.

\section{RESULTS}

Initially the penetration depth for the scheme is measured. It gives a penetration depth of $14 \mathrm{~cm}(0 \mathrm{~dB}$ signal-to-noise ratio) when using a tissue mimicking phantom with an attenuation of $0.5 \mathrm{~dB} /[\mathrm{MHz} \mathrm{cm}]$. The SA imaging sequence and array were also simulated in Field II $[20,21]$ and yielded a PSF with a size of $(1.17 \lambda \times 2.12 \lambda \times 0.63 \lambda)$ at $15 \mathrm{~mm}$.

The resulting 3-D SR image is shown in Fig. 1, where each blue dot indicates the identification of a bubble. The full geometry of the phantom can be seen with the inlet and outlet and the detected bubbles seem confined to the tube.

The localization in the $y-z$ has been investigated by selecting the bubble only moving in the $x$ direction as is shown in the top graph in Fig. 2, where blue crosses are the selected bubbles and red dots indicates all localized bubbles. Lines have then been fitted to the center of all the locations as shown in Fig. 3, so the distance from the tube center to the bubble locations can be found. The radial positions are then found and shown in Fig. 4. Bubbles inside the tube are marked by a cross and bubbles outside are marked by a red circle with a blue cross.

The fraction of bubbles outside the tube, as shown in Fig. 5, is then an indication of the precision of the bubble localization as described in Section II-D. The fraction is in this case $13.0 \%$, which translates to a precision of $16.5 \mu \mathrm{m}$. The fraction is $18.2 \%$ in the $x-z$ plane translating to a precision of $23.0 \mu \mathrm{m}$. The simulated point spread function of the imaging setup at this depth is $0.58 \times 1.05 \times 0.31 \mathrm{~mm}^{3}$ $(x, y, z)$, which corresponds to an interrogated volume of 0.189 $\mathrm{mm}^{3}$. Assuming the precision in all three coordinates is 23.0 $\mu \mathrm{m}$ gives a volume of $12,167 \mu \mathrm{m}^{3}$, which is 15,700 times smaller than for the PSF limited system.

\section{Discussion AND CONCLUSION}

A 3-D SR measurement scheme and processing pipeline have been presented. The approach uses a $62+62$ elements RC probe, where only rows are used for emission and columns for reception. The scheme employs two times 32 emissions for pulse inversion imaging attaining a volume rate of 240
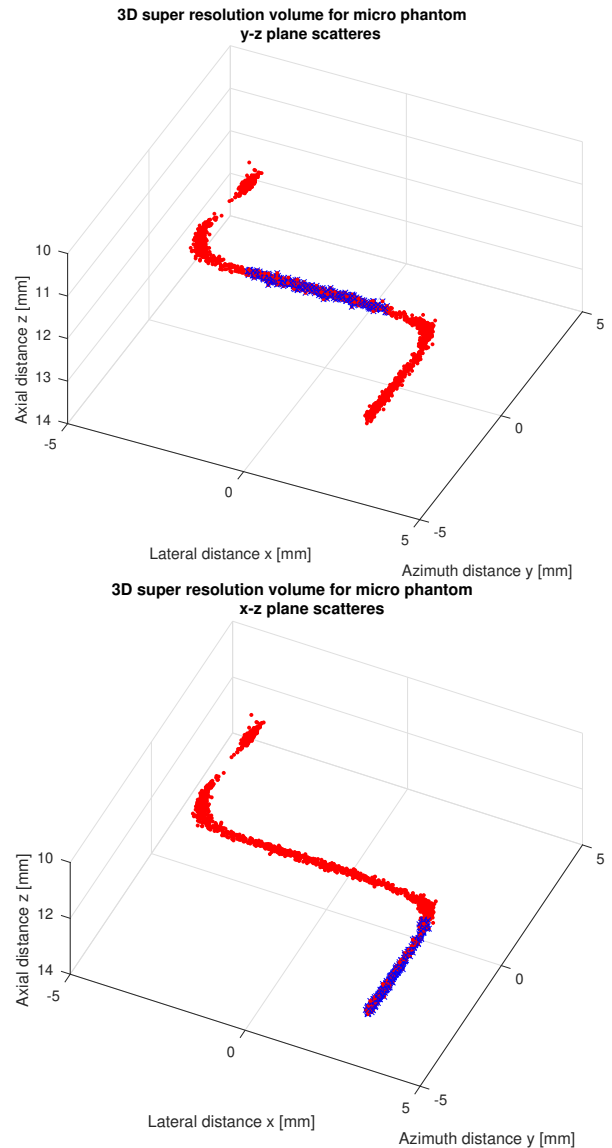

Fig. 2. Identification of bubbles only flowing in the $x$ direction (top) and only in the $y$ direction in the outlet (bottom). Red dots show all the bubble locations and blue crosses are the selected bubbles.
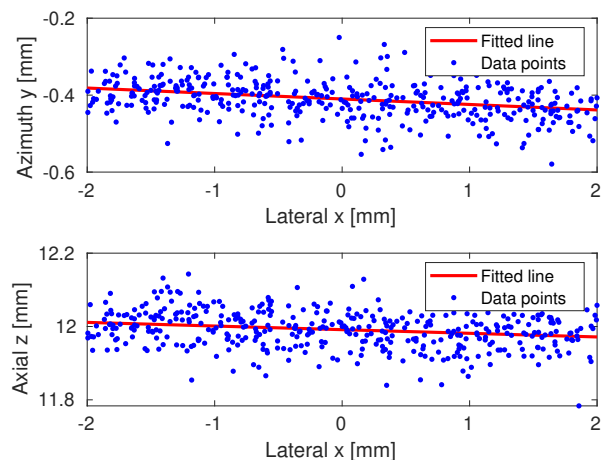

Fig. 3. Fitted center line for the bubble locations with movement in the $x$ direction.

$\mathrm{Hz}$ down to $5 \mathrm{~cm}$ or $85 \mathrm{~Hz}$ down to $14 \mathrm{~cm}$, which is the penetration depth of the imaging scheme.

The major advantage is that the volume is focused in all three directions including the elevation direction, which yields a resolution of $(1.17 \lambda \times 2.12 \lambda \times 0.63 \lambda)$ at a depth of 15 $\mathrm{mm}$. This was attained for a modest 62 elements, which both reduces the amount of data from the probe by a factor of 8 compared to previous 3-D SRI [13] as well as the beamforming time for a probe with 4 times the area of a 1024 elements 2-D matrix probe. 


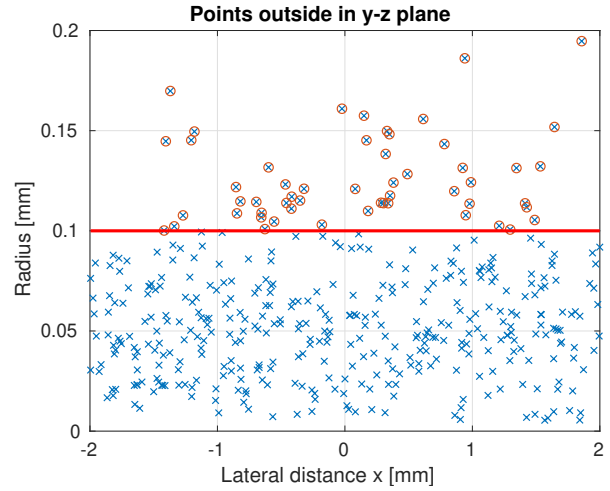

Fig. 4. Radius of the bubble position relative to the fitted center line in the $y-z$ plane. Blue crosses marks the locations of all bubbles and red circles mark bubbles outside the vessel boundary.

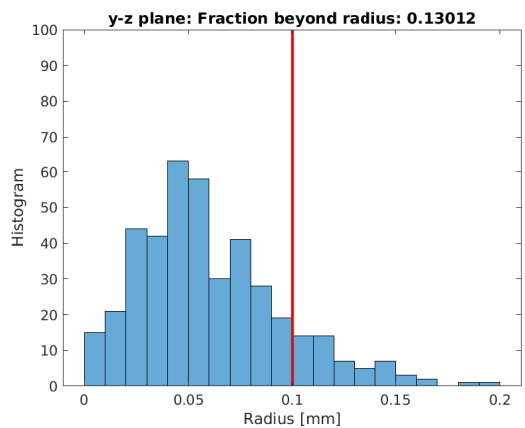

Fig. 5. Histogram for the bubble radius. The red line indicates the $100 \mu \mathrm{m}$ radius of the tube and the fraction of bubbles outside is $13.0 \%$.

The processing pipeline yielded a precision of $16.5 \mu \mathrm{m}$ in the $y-z$ plane and $23.0 \mu \mathrm{m}$ in the $x-z$ plane, which is 15,700 times smaller in volume than for the PSF limited $\mathrm{RC}$ system. The number of active elements is three times less than what is available in commercial ultrasound consoles, and the beamforming can be attained in near real-time, when employing a state-of-the-art GPU card [22].

\section{ACKNOWLEDGEMENT}

This work was financially supported by grant 7050-00004B from Innovation Fund Denmark, and from BK Medical, Herlev, Denmark.

\section{REFERENCES}

[1] O. Couture, B. Besson, G. Montaldo, M. Fink, and M. Tanter, "Microbubble ultrasound super-localization imaging (MUSLI)," in Proc. IEEE Ultrason. Symp., 2011, pp. 1285-1287.

[2] O. M. Viessmann, R. J. Eckersley, K. C. Jeffries, M. X. Tang, and C. Dunsby, "Acoustic super-resolution with ultrasound and microbubbles," Phys. Med. Biol., vol. 58, pp. 6447-6458, 2013.

[3] M. Siepmann, G. Schmitz, J. Bzyl, M. Palmowski, and F. Kiessling, "Imaging tumor vascularity by tracing single microbubbles," Proc. IEEE Ultrason. Symp., pp. 6293 297, 1906-1908, 2011.

[4] M. A. O'Reilly and K. Hynynen, "A super-resolution ultrasound method for brain vascular mapping," Med. Phys., vol. 40, no. 11, pp. 110701-7, 2013.

[5] K. Christensen-Jeffries, R. J. Browning, M. Tang, C. Dunsby, and R. J. Eckersley, "In vivo acoustic super-resolution and super-resolved velocity mapping using microbubbles," IEEE Trans. Med. Imag., vol. 34, no. 2, pp. 433-440, February 2015.
[6] C. Errico, J. Pierre, S. Pezet, Y. Desailly, Z. Lenkei, O. Couture, and M. Tanter, "Ultrafast ultrasound localization microscopy for deep superresolution vascular imaging," Nature, vol. 527, pp. 499-502, November 2015.

[7] O. Couture, V. Hingot, B. Heiles, P. Muleki-Seya, and M. Tanter, "Ultrasound localization microscopy and super-resolution: A state of the art," IEEE Trans. Ultrason., Ferroelec., Freq. Contr., vol. 65, no. 8, pp. 1304-1320, 2018.

[8] J. Zhu, E. M. Rowland, S. Harput, K. Riemer, C. H. Leow, B. Clark, K. Cox, A. Lim, K. Christensen-Jeffries, G. Zhang, J. Brown, C. Dunsby, R. J. Eckersley, P. D. Weinberg, and M.-X. Tang, "3D super-resolution US imaging of rabbit lymph node vasculature in vivo by using microbubbles," Radiology, vol. 291, no. 3, pp. 642-650, 2019.

[9] F. Lin, S. E. Shelton, D. Espindola, J. D. Rojas, G. Pinton, and P. A. Dayton, "3-D ultrasound localization microscopy for identifying microvascular morphology features of tumor angiogenesis at a resolution beyond the diffraction limit of conventional ultrasound," Theranostics, vol. 7, no. 1, pp. 196-204, 2017.

[10] K. Christensen-Jeffries, S. Harput, J. Brown, P. N. T. Wells, P. Aljabar, C. Dunsby, M. Tang, and R. J. Eckersley, "Microbubble axial localization errors in ultrasound super-resolution imaging," IEEE Trans. Ultrason., Ferroelec., Freq. Contr., vol. 64, no. 11, pp. 1644-1654, 2017.

[11] J. A. Jensen, H. Holten-Lund, R. T. Nilsson, M. Hansen, U. D. Larsen, R. P. Domsten, B. G. Tomov, M. B. Stuart, S. I. Nikolov, M. J. Pihl, Y. Du, J. H. Rasmussen, and M. F. Rasmussen, "SARUS: A synthetic aperture real-time ultrasound system," IEEE Trans. Ultrason., Ferroelec., Freq. Contr., vol. 60, no. 9, pp. 1838-1852, 2013.

[12] L. Petrusca, F. Varray, R. Souchon, A. Bernard, J. Y. Chapelon, H. Liebgott, W. A. N'Djin, and M. Viallon, "Fast volumetric ultrasound B-mode and Doppler imaging with a new high-channels density platform for advanced 4D cardiac imaging/therapy," Applied Sciences (Switzerland), vol. 8, no. 2, pp. 200:1-15, 2018.

[13] S. Harput, K. Christensen-Jeffries, A. Ramalli, J. Brown, J. Zhu, G. Zhang, C. H. Leow, M. Toulemonde, E. Boni, P. Tortoli, R. J. Eckersley, C. Dunsby, and M. Tang, "3-D super-resolution ultrasound (SR-US) imaging with a 2-D sparse array," arXiv preprint, p. 1902.01608v1, 2019.

[14] E. Boni, L. Bassi, A. Dallai, F. Guidi, V. Meacci, A. Ramalli, S. Ricci, and P. Tortoli, "ULA-OP 256: A 256-channel open scanner for development and real-time implementation of new ultrasound methods," IEEE Trans. Ultrason., Ferroelec., Freq. Contr., vol. 63, no. 10, pp. 14881495,2016

[15] M. Engholm, H. Bouzari, T. L. Christiansen, C. Beers, J. P. Bagge, L. N. Moesner, S. E. Diederichsen, M. B. Stuart, J. A. Jensen, and E. V. Thomsen, "Probe development of CMUT and PZT row-columnaddressed 2-D arrays," Sens. Actuators A: Phys., vol. 273, pp. 121-133, 2018.

[16] M. F. Rasmussen, T. L. Christiansen, E. V. Thomsen, and J. A. Jensen, "3-D imaging using row-column-addressed arrays with integrated apodization - Part I: Apodization design and line element beamforming," IEEE Trans. Ultrason., Ferroelec., Freq. Contr., vol. 62, no. 5, pp. 947-958, 2015

[17] H. Bouzari, M. Engholm, S. I. Nikolov, M. B. Stuart, E. V. Thomsen, and J. A. Jensen, "Imaging performance for two row-column arrays," IEEE Trans. Ultrason., Ferroelec., Freq. Contr., vol. 66, no. 7, pp. 1209-1221, 2019.

[18] M. L. Ommen, M. Schou, R. Zhang, C. A. V. Hoyos, J. A. Jensen, N. B. Larsen, and E. V. Thomsen, "3D printed flow phantoms with fiducial markers for super-resolution ultrasound imaging," in Proc. IEEE Ultrason. Symp., 2018, pp. 1-4.

[19] M. B. Stuart, M. Schou, and J. A. Jensen, "Row-column beamforming with dynamic apodizations on a GPU," in Proc. SPIE Med. Imag., 2019, pp. 1-7, paper number 10955-20.

[20] J. A. Jensen and N. B. Svendsen, "Calculation of pressure fields from arbitrarily shaped, apodized, and excited ultrasound transducers," IEEE Trans. Ultrason., Ferroelec., Freq. Contr., vol. 39, no. 2, pp. 262-267, 1992.

[21] J. A. Jensen, "Field: A program for simulating ultrasound systems," Med. Biol. Eng. Comp., vol. 10th Nordic-Baltic Conference on Biomedical Imaging, Vol. 4, Supplement 1, Part 1, pp. 351-353, 1996.

[22] M. B. Stuart, P. M. Jensen, J. T. R. Olsen, A. B. Kristensen, M. Schou, B. Dammann, H. H. B. Sørensen, and J. A. Jensen, "Fast GPUbeamforming of row-column addressed probe data," in Proc. IEEE Ultrason. Symp., 2019, pp. 1-4. 